\title{
CÁC YẾU TỐ TÁC ĐộNG ĐẾN NHận THỬC VÀ THÁI Độ CỦA KHÁCH HÀNG ĐỐI VỚI HOẠT ĐỘNG TRÁCH NHIẸM XÃ HỘI DÖANH NGHIỆP
}

\author{
NGUYỄN NGỌC HIỀN, NGUYỄN TRỌNG MINH THÁI \\ Truờng Đại học Công nghiệp Thành phố Hồ Chí Minh \\ nguyenngochien.qn@iuh.edu.vn,nguyentrongminhthai@iuh.edu.vn
}

Tóm tắt. Các nghiên cứu về nhận thức và thái độ của khách hàng đối với hoạt động trách nhiệm xã hội doanh nghiệp (CSR) đã nhận được nhiều sự quan tâm trong những năm gần đây. Tuy nhiên, dòng nghiên cứu về tiền đề của nhận thức và thái độ của khách hàng đối với CSR còn mới mẻ. Nghiên cứu này đề xuất và kiểm định mô hình trong đó hình ảnh nước xuất xứ, danh tiếng doanh nghiệp và niềm tin của khách hàng được xem là tiền đề của nhận thức và thái độ của khách hàng đối với CSR. Mô hình được kiểm định thông qua mẫu 615 khách hàng sở hữu xe ô tô du lịch. Kết quả cho thấy, hình ảnh nước xuất xứ thương hiệu, hình ảnh nước sản xuất, danh tiếng và niềm tin đều có ảnh hưởng tích cực đến nhận thức CSR. Ngoài ra, nhận thức CSR là trung gian tác động của hình ảnh nước xuất xứ thương hiệu và niềm tin đến thái độ của khách hàng. Các hàm ý của nghiên cứu được thảo luận và bài báo cũng cung cấp những khuyến nghị cho các nghiên cứu trong tương lai.

Tù khóa. Hình ảnh nước xuất xứ, danh tiếng, niềm tin, trách nhiệm xã hội doanh nghiệp, thái độ của khách hàng.

\section{FACTORS AFFECTING CUSTOMER PERCEPTION AND ATTITUDE TOWARD CORPORATE SOCIAL RESPONSIBILITY}

\begin{abstract}
Studies on customer perception and attitude toward Corporate Social Responsibility (CSR) have received a lot of attention in recent years. However, the research on the antecedent of customer perception and attitude toward CSR is still relatively new. This study proposes and tests the model, in which the country-of-origin, the corporate reputation and the customer trust are considered as the antecedent of customer perception and attitude toward CSR. By using the simple of 615 customers, who owned passenger cars, the results show that the country-of-brand image, country-of-manufacture image, reputation and trust have positive impact on perceived CSR. In addition, perceived CSR is the mediator factor contributing to the effect of the country-of-brand image and customer trust on customer attitudes. The implications of the study are discussed and this paper also provides recommendations for future research.
\end{abstract}

Keywords. Country-of-origin; Reputation; Trust; Corporate social responsibility; Customer attitude.

\section{GIỚI THIỆU}

Trách nhiệm xã hội doanh nghiệp (CSR) đề cập đến nghĩa vụ đạo đức và nghĩa vụ xã hội của doanh nghiệp vượt ra ngoài lợi ích kinh tế riêng có của nó [1]. Do những mối quan tâm ngày càng tăng về việc thực hiện CSR của các doanh nghiệp, trong những năm gần đây CSR đã trở thành thực tiễn kinh doanh quan trọng trên toàn cầu vì danh tiếng và cải thiện hoạt động tài chính của doanh nghiệp [2]. Các nghiên cứu hiện tại tập trung xem xét tác động của CSR đối với hoạt động tài chính của doanh nghiệp, phần lớn các nghiên cứu cho thấy những kết quả tích cực đối với hoạt động CSR. Các nghiên cứu về phản hồi của người tiêu dùng đối với CSR còn hạn chế [3]. Hơn nữa, các nghiên cứu hiện tại tập trung vào cách khách hàng nhận thức các hoạt động CSR, cụ thể thử nghiệm một hành động liên quan đển CSR nhằm nỗ lực xác định ảnh hưởng của nó đến nhận thức của khách hàng [4]. Tuy nhiên, một số nhà nghiên cứu đặt câu hỏi liệu các hoạt động CSR có luôn luôn dẫn đến hoạt động kinh doanh tốt hơn, vì công chúng thường xem các hoạt động đó như là hoạt động tư lợi [5]. Như vậy, những yếu tố nào dẫn đến nhận thức CSR của khách hàng cần được xem xét. Ngoài ra, nghiên cứu trước cho thấy người tiêu dùng có thái độ tốt hơn đối với các doanh nghiệp có trách nhiệm đối với xã hội, tuy nhiên vẫn không có sự thống nhất trong phản ứng của người tiêu dùng [6]. 
Theo đó, nghiên cứu này nhằm khám phá những yếu tố ảnh hưởng đến nhận thức và thái độ của khách hàng đối với hoạt động CSR. Mô hình được kiểm định trong bối cảnh khách hàng ngành ô tô tại thị trường Việt Nam, một thị trường tiêu thụ tiềm năng lớn đối với các thương hiệu ô tô toàn cầu.

\section{CƠ SỞ LÝ THUYẾT VÀ MÔ HÌNH NGHIÊN CÚU}

\subsection{Cơ sở lí thuyết và giả thuyết nghiên cứu}

\subsubsection{Trách nhiệm xã hội doanh nghiệp (CSR - Corporate Social Responsibility)}

Trách nhiệm xã hội của doanh nghiệp đã trở nên phổ biến vào đầu thế kỷ 21 . Khách hàng đang yêu cầu các doanh nghiệp thực hiện CSR bất kể quy mô, loại hình và nguồn gốc của các doanh nghiệp. Vì các quốc gia gặp nhiều vấn đề về xã hội, nên khách hàng mong đợi các doanh nghiệp giải quyết những vấn đề này bằng cách sử dụng các nguồn lực của họ một cách hiệu quả [7]. Carroll (1979) [8] cho rằng có thể phân biệt các loại nghĩa vụ xã hội khác nhau: (a) Nghĩa vụ về kinh tế (hiệu quả về mặt kinh tế), (b) nghĩa vụ pháp lý và đạo đức (tuân theo quy định của pháp luật và các giá trị chuẩn mực được thừa nhận) và (c) nghĩa vụ từ thiện (chủ động đóng góp cho xã hội). CSR là một công cụ tiếp thị quan trọng của doanh nghiệp bởi vì nó đáp ứng được kỳ vọng của người tiêu dùng, cải thiện hiệu suất và danh tiếng doanh nghiệp. CSR ngày càng nhận được sự quan tâm của các nhà nghiên cứu. Tuy nhiên, các nghiên cứu về CSR trong thời gian gần đây chủ yếu tập trung vào việc xác định mối quan hệ giữa CSR và hiệu suất của tổ chức. Rất ít nghiên cứu chứng minh điều gì thực sự tạo ra hoặc thúc đẩy nhận thức CSR [5].

\subsubsection{Nhận thức và thái độ của khách hàng đối với hoạt động trách nhiệm xã hội doanh nghiệp}

Các tài liệu về CSR đã nổi lên từ hai quan điểm. Một dòng nghiên cứu đến từ các tài liệu quản lý, trọng tâm ở đây là câu hỏi liệu doanh nghiệp có nên tham gia vào các hoạt động CSR không và thực hiện những hoạt động CSR có ảnh hưởng như thế nào đến hoạt động tài chính. Một dòng nghiên cứu từ tài liệu tiếp thị tập trung vào cách khách hàng nhận thức các hoạt động CSR, cụ thể là thử nghiệm một hành động liên quan đến CSR và xem xét phản ứng của khách hàng nhằm nỗ lực xác định ảnh hưởng của CSR [4]. Cả hai quan điểm đã đề cập đến chủ đề này trong nhiều thập kỷ qua. Nghiên cưu này xem xét cách khách hàng nhận thức các hoạt động trách nhiệm xã hội doanh nghiệp.

Thái độ được định nghĩa là cảm xúc tích cực hay tiêu cực của một người về một hành động nói chung. Thái độ cho thấy những cảm xúc bền vững và khuynh hướng hướng tới một sản phẩm hay một ý tưởng [9]. Thái độ của khách hàng có thể bị ảnh hưởng bởi hành vi đạo đức của doanh nghiệp. Nhận thức CSR ảnh hưởng đến thái độ của khách hàng và thái độ sẽ ảnh hưởng đến ý định và hành vi của họ [10]. Nghiên cứu của Sen và cộng sự (2006) [11] cũng cho thấy có mối quan hệ giữa nhận thức CSR và thái độ của khách hàng. Như vậy có thể đưa ra giả thuyết như sau:

Giả thuyết 1: Nhận thức trách nhiệm xã hội doanh nghiệp có tác động tích cực đến thái độ của khách hàng.

\subsubsection{Hình ảnh nước xuất xứ}

Hình ảnh nước xuất xứ được xác định bởi Roth và Romeo (1992) [12] như tổng thể nhận thức của khách hàng được hình thành về sản phẩm từ một quốc gia cụ thể, dựa trên kinh nghiệm trước đây của họ về sản xuất, tiếp thị, điểm mạnh và điểm yếu của sản phẩm. Gardberg và Schepers (2008) [13] đã tiến hành nghiên cứu thực nghiệm tại Mỹ, kết quả cho thấy rằng, nhận thức của công chúng về CSR đối với các doanh nghiệp nước ngoài thấp hơn các doanh nghiệp trong nước. Trong khi đó, nghiên cứu của Han (2015) [14] cho rằng người tiêu dùng Hàn Quốc kỳ vọng lớn hơn đối với các doanh nghiệp đến từ Châu âu so với các doanh nghiệp Hàn Quốc liên quan đến trách nhiệm pháp lý, kinh tế và đạo đức. Ngoài ra, Nước xuất xứ cũng nổi lên như là dự báo có ý nghĩa về thái độ của khách hàng đối với các hoạt động trách nhiệm xã hội doanh nghiệp [13]. Như vậy giả thuyết được đưa ra như sau:

Giả thuyết 2a: Hình ảnh nước xuất xứ thương hiệu có tác động tích cực đến nhận thức trách nhiệm xã hội doanh nghiệp.

Giả thuyết 2b: Hình ảnh nước xuất xứ thương hiệu có tác động tích cực đến thái độ của khách hàng. 
ĐỐI VỚI HOẠT ĐộNG TRÁCH NHIỆM XÃ HỘI DOANH NGHIỆP

Giả thuyết 2c: Nhận thức trách nhiệm xã hội doanh nghiệp là trung gian tác động của hình ảnh nước xuất xứ thương hiệu đến thái độ của khách hàng.

Ngoài ra, với sự phát triển của các tổ chức đa quốc gia, cùng với sự đầu tư trực tiếp nước ngoài lớn vào các nước đang phát triển, với mục tiêu cắt giảm chi phí hoạt động và tận dụng mức lương thấp hơn, đã thúc đẩy sự xuất hiện của các sản phẩm và thương hiệu lai ghép. Nước xuất xứ không còn có thể được xem xét như là một khái niệm duy nhất, thay vào đó nó cần được nghiên cứu như là một khái niệm đa chiều [15]. Trong đó, ngoài khái niệm nước xuất xứ thương hiệu thì nước sản xuất cũng được xem xét chính, bởi vì nó ảnh hưởng mạnh mẽ đến nhận thức và đánh giá của khách hàng [16]. Và giả thuyết được đưa ra như sau:

Giả thuyết 3: Hình ảnh nước sản xuất có tác động tích cực đến nhận thức trách nhiệm xã hội doanh nghiệp.

\subsubsection{Danh tiếng doanh nghiẹp}

Fombrun (1996) [17] xác định danh tiếng là nhận thức các hành động quá khứ của một doanh nghiệp và mô tả triển vọng trong tương lai của tổng thể doanh nghiệp cho tất cả các bên quan trọng của nó khi so sánh với các đối thủ hàng đầu khác. Bae và Cameron (2006) [18] khẳng định rằng công chúng có xu hướng suy luận hoạt động từ thiện của một doanh nghiệp như một hoạt động cùng có lợi khi một công ty có danh tiếng tốt. Tuy nhiên, khi một doanh nghiệp có danh tiếng xấu, mọi người có xu hướng suy luận hoạt động từ thiện của công ty như là một hoạt động tư lợi. Kết quả nghiên cứu của Shim và Yang (2016) [5] cho thấy, nhận thức về đạo đức giả của doanh nghiệp là trung gian tác động của danh tiếng đến thái độ đối với một doanh nghiệp trong khủng hoảng. Nghiên cứu cho thấy rằng CSR được sử dụng tốt nhất trong điều kiện danh tiếng doanh nghiệp tốt và không có khủng hoảng. Như vậy có thể đưa ra giả thuyết như sau:

Giả thuyết 4: Danh tiếng doanh nghiệp có tác động tích cực đến nhận thức trách nhiệm xã hội doanh nghiệp.

\subsubsection{Niềm tin của khách hàng}

Niềm tin đã được hiểu như sự tin tưởng rằng doanh nghiệp sẽ thực hiện một cách phù hợp với mong đợi của họ [19]. Nghiên cứu cho thấy một mối liên hệ tích cực giữa các hoạt động CSR và thái độ của khách hàng đối với các doanh nghiệp [20]. Tuy nhiên, vẫn chưa có nghiên cứu tác động của niềm tin đối với nhận thức trách nhiệm xã hội doanh nghiệp. Theo Mayer và cộng sự (1995) [21] niềm tin bao gồm niềm tin chuyên môn, niềm tin chính trực và niềm tin lòng nhân từ xã hội. Niềm tin chuyên môn là sự tin tưởng của người tiêu dùng rằng một doanh nghiệp có khả năng và kỹ năng kỹ thuật để sản xuất và cung cấp sản phẩm cụ thể đáp ứng nhu cầu của người tiêu dùng. Niềm tin chính trực là sự tin tưởng rằng một doanh nghiệp thể hiện sự nhất quán giữa các giá trị và hành vi của họ. Và cuối cùng, niềm tin nhân từ xã hội đề cập đến niềm tin rằng một doanh nghiệp thực sự quan tâm đến việc giữ gìn và nâng cao phúc lợi của xã hội [22]. Khi khách hàng có niềm tin đối với một doanh nghiệp, họ sẽ cho rằng doanh nghiệp đó tuân thủ các nguyên tắc đạo đức, quan tâm đến phúc lợi xã hội [22], dẫn đến nhận thức và thái độ tích cực đối với hoạt động trách nhiệm xã hội của doanh nghiệp. Dựa trên quan điểm này, giả thuyết được đưa ra như sau:

Giả thuyết 5a: Niềm tin của khách hàng có tác động tích cực đến nhận thức trách nhiệm xã hội doanh nghiệp.

Giả thuyết 5b: Niềm tin của khách hàng có tác động tích cực đến thái độ của khách hàng.

Giả thuyết 5c: Nhận thức trách nhiệm xã hội là trung gian tác động của niềm tin đến thái độ của khách hàng. 


\subsection{Mô hình nghiên cứu}

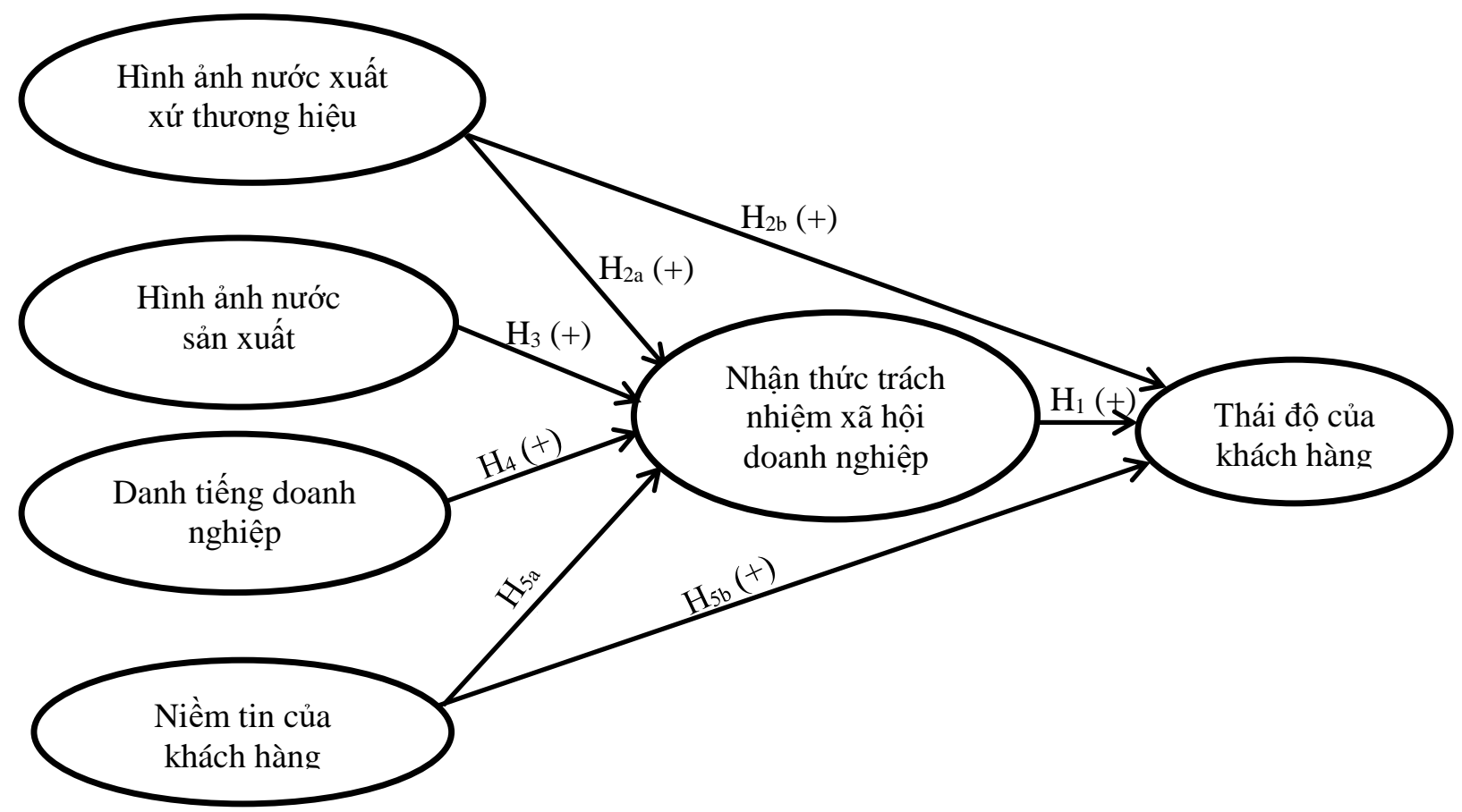

Hình 1. Mô hình nghiên cứu đề xuất

Nguồn: Tác giả đề xuất

\section{PHƯƠNG PHÁP NGHIÊN CÚU}

\section{1. Đo lường}

Thang đo lường nhận thức trách nhiệm xã hội doanh nghiệp được kế thừa từ thang đo của Singh và cộng sự (2008) [23]. Tuy nhiên, thang đo này còn tương đối với tại Việt Nam. Vì vậy, tác giả tiến hành thảo luận nhóm (08 khách hàng sở hữu xe ô tô du lịch). Kết quả cho thấy, ngoài những biến tương tự như thang đo đã lựa chọn, khách hàng cho rằng nên bổ sung các biến: "Luôn nổ lực cải thiện sản phẩm và dịch vụ cho khách hàng", "Thông báo chính xác và trung thực về đặc điểm sản phẩm" và "Doanh nghiệp trung thực trong hoạt động quảng cáo/khuyến mãi”. Kết quả hình thành thang đo lường nhận thức CSR gồm 3 thành phần: trách nhiệm thương mại, trách nhiệm đạo đức và trách nhiệm xã hội và 14 biến. Ngoài ra, thang đo lường danh tiếng doanh nghiệp dựa trên thang đo của Ponzi và cộng sự (2011) [24] cũng được điều chỉnh thông qua thảo luận nhóm. Khách hàng cho rằng nên bổ sung biến "Công chúng đánh giá tốt về doanh nghiệp này". Kết quả hình thành thang đo danh tiếng doanh nghiệp gồm 5 biến.

Hình ảnh nước xuất xứ thương hiệu sử dụng thang đo của Yasin và cộng sự (2007) [25] gồm 7 biến. Hình ảnh nước sản xuất sử dụng thang đo của Chen và Su (2011) [26] gồm 4 biến. Niềm tin của khách hàng được đo lường bằng thang đo của Keh và Xie (2009) [27] gồm 5 biến. Và cuối cùng, thang đo lường thái độ của khách hàng đối với CSR được đo lường bằng thang đo Sun và Wang (2010) [28] gồm 3 biến.

\subsection{Thu thập dữ liệu}

Nghiên cứu sử dụng kỹ thuật lấy mẫu thuận tiện. Ngoài ra, căn cứ tỷ lệ sở hữu xe ô tô theo vùng miền: miền nam chiếm $34 \%$, miền trung chiếm $19 \%$ và miền bắc chiếm $34 \%$ trong tổng số gần 1,3 triệu xe được sở hữu tại Việt Nam. Nghiên cứu này chọn mẫu theo tỷ lệ trên giữa các vùng miền. Nghiên cứu được thực hiện từ tháng 09/2017 đến tháng 12/2017. Phỏng vấn trực tiếp đối với khách hàng sở hữu và sử dụng ô tô du lịch tại TP.HCM và Đà Nẵng. Tổng số phiếu điều tra là 800 phiếu, tổng số phiếu thu về và hợp lệ là 615 phiếu được chọn làm dữ liệu cho nghiên cứu này. 


\section{PHÂN TÍCH DŨ̉ LIÊ̂U VÀ KẾT QUẢ}

\section{1. Đặc điểm mẫu thống kê}

Bảng 1. Cơ cấu mẫu nghiên cứu

\begin{tabular}{|c|c|c|c|c|c|c|c|}
\hline \multicolumn{2}{|r|}{ Đặc điểm } & \multirow{2}{*}{$\begin{array}{c}\text { Số lượng } \\
293 \\
\end{array}$} & \multirow{2}{*}{$\begin{array}{l}\text { Tỷ lệ } \\
(\%)\end{array}$} & \multicolumn{2}{|c|}{ Đặc điểm } & \multirow{2}{*}{$\begin{array}{c}\begin{array}{c}\text { Số } \\
\text { lượng }\end{array} \\
535\end{array}$} & \multirow{2}{*}{$\begin{array}{l}\text { Tỷ lệ } \\
\text { (\%) }\end{array}$} \\
\hline \multirow{3}{*}{ Quê quán } & Miền Bắc & & & \multirow{2}{*}{$\begin{array}{l}\text { Giới } \\
\text { tính }\end{array}$} & Nam & & \\
\hline & Miền Trung & 114 & 18,5 & & Nũ̃ & 80 & 13,0 \\
\hline & Miền Nam & 208 & 33,8 & \multirow{6}{*}{$\begin{array}{c}\text { Thương } \\
\text { hiệu } \\
\text { xe }\end{array}$} & Mazda & 72 & 11,7 \\
\hline \multirow{5}{*}{ Tuổi } & $18-25$ & 50 & 8,1 & & Kia & 90 & 14,6 \\
\hline & $26-35$ & 180 & 29,3 & & Toyota & 220 & 35,8 \\
\hline & $36-45$ & 196 & 31,9 & & Ford & 93 & 15,1 \\
\hline & $46-55$ & 145 & 23,6 & & Honda & 65 & 10,6 \\
\hline & $56-65$ & 44 & 7,2 & & Loai khác & 75 & 12,2 \\
\hline \multirow{5}{*}{$\begin{array}{l}\text { Trình độ } \\
\text { học vấn }\end{array}$} & Tiểu học & 3 & 0,5 & \multirow{5}{*}{$\begin{array}{c}\text { Nước } \\
\text { xuất xứ }\end{array}$} & Nhật Bản & 372 & 60,5 \\
\hline & Trung học cơ sở & 45 & 7,3 & & Mỹ & 106 & 17,2 \\
\hline & Trung học phổ thông & 155 & 25,2 & & Đức & 15 & 2,4 \\
\hline & Đại học & 266 & 43,3 & & Hàn Quốc & 109 & 17,7 \\
\hline & Sau đại học & 146 & 23,7 & & Nước khác & 13 & 2,1 \\
\hline \multirow{5}{*}{ Thu nhập } & Dưới 10 triệu đồng & 95 & 15,4 & \multirow{5}{*}{$\begin{array}{c}\text { Nước } \\
\text { sản xuất }\end{array}$} & Thái Lan & 57 & 9,3 \\
\hline & 10 - 15 triệu đồng & 156 & 25,4 & & Indonesia & 48 & 7,8 \\
\hline & 15 - 20 triệu đồng & 110 & 17,9 & & Việt Nam & 312 & 50,7 \\
\hline & 20 - 25 triệu đồng & 104 & 16,9 & & Trung Quốc & 57 & 9,3 \\
\hline & Trên 25 triệu đồng & 150 & 24,4 & & Nước khác & 141 & 22,9 \\
\hline
\end{tabular}

(Nguồn: Kết quả xủ lý số liệu trên phần mềm SPSS 24.0)

Đặc điểm mẫu thống kê (Bảng 1) cho thấy, tỷ lệ quê quán của những người trả lời phù hợp với tỷ lệ sở hữu xe ô tô giữa miền bắc, miền trung và miền nam Việt Nam. Ngoài ra, mẫu nghiên cứu có đặc điểm chủ yếu là: có giới tính chủ yếu là nam, độ tuổi phổ biến từ 26 đến 55 tuổi, có trình độ chủ yếu từ trung học phổ thông trở lên, có thu nhập phân bố tương đối đồng đều từ 10 triệu đồng đến trên 25 triệu đồng. Thương hiệu xe ô được khảo sát nhiều nhất là Toyota, nước xuất xứ thương hiệu nhiều nhất là Nhật Bản và nước sản xuất nhiều nhất là Việt Nam.

\subsection{Kiểm định thang đo}

\subsection{1. Đánh giá độ tin cậy thang đo}

Kết quả kiểm định độ tin cậy các thang đo trong mô hình nghiên cứu cho thấy, tất cả thang đo thành phần đều có hệ số Cronbach alpha lớn hơn 0,6 [29], đảm bảo độ tin cậy cần thiết. Tuy nhiên, biến CR4 có hệ số tương quan biến tổng là 0,257 nhỏ hơn 0,3 và biến ER1 có hệ số tương quan biến tổng là 0,242 nhỏ hơn 0,3 nên loại các biến này [30]. Kết quả kiểm định sau khi loại biến, các biến còn lại của các thang đo trong mô hình nghiên cứu đều đạt độ tin cậy cần thiết.

\subsection{2. Đánh giá giá trị của thang đo - phân tích nhân tố khám phá (EFA)}

Phép trích nhân tố Principal Axis Factoring $(\mathrm{PAF})$ và phép quay không vuông góc Promax được sử dụng. Kết quả phân tích EFA cho thấy, hệ số $\mathrm{KMO}=0.883>0,5$ và $\mathrm{Sig}(\mathrm{Kiểm} \mathrm{định} \mathrm{Bartlett})=0,00<0,05$, cho thấy phân tích EFA là phù hợp. 
Bảng 2. Trọng số nhân tố của thang đo

\begin{tabular}{|c|c|}
\hline Khái niệm và biến quan sát & $\begin{array}{c}\text { Trọng số } \\
\text { nhân tố }\end{array}$ \\
\hline \multicolumn{2}{|l|}{ Danh tiếng doanh nghiệp (CREP) } \\
\hline CREP1. Tôi có cảm nhận tốt về doanh nghiệp & 0,804 \\
\hline CREP2. Tôi tin tưởng doanh nghiệp & 0,868 \\
\hline CREP3. Tôi ngưỡng mộ và tôn trọng doanh nghiệp & 0,647 \\
\hline CREP4. Là doanh nghiệp có danh tiếng tốt & 0,712 \\
\hline CREP5. Công chúng đánh giá tốt về doanh nghiệp này & 0,665 \\
\hline \multicolumn{2}{|l|}{ Hình ảnh nước xuất xứ thương hiệu (COBI) } \\
\hline COBI1. Nước sáng tạo trong sản xuất & 0,660 \\
\hline COBI2. Nước có trình độ công nghệ cao & 0,879 \\
\hline COBI3. Nước có thiết kế tốt & 0,567 \\
\hline COBI4. Là nước có tay nghề sáng tạo & 0,716 \\
\hline COBI5. Là nước có tay nghề chất lượng cao & 0,636 \\
\hline COBI6. Là nước có uy tín & 0,613 \\
\hline COBI7. Là nước tiên tiến & 0,751 \\
\hline \multicolumn{2}{|l|}{ Hình ảnh nước sản xuất (COMI) } \\
\hline COMI1. Người dân nước này có tay nghề xuất sắc & 0,747 \\
\hline COMI2. Người dân nước này đáng tin cậy & 0,805 \\
\hline COMI3. Sản phẩm sản xuất ở nước này bền & 0,894 \\
\hline COMI4. Sản phầm sản xuât ở nước này có chất lượng cao & 0,845 \\
\hline \multicolumn{2}{|l|}{ Niềm tin của khách hàng (TRUST) } \\
\hline TRUST1. Doanh nghiệp có đủ năng lực để thực hiện công việc & 0,783 \\
\hline TRUST2. Doanh nghiệp đáng tin cậy & 0,906 \\
\hline TRUST3. Doanh nghiệp trung thực và có những nguyên tắc đạo đức mạnh mẽ & 0,753 \\
\hline TRUST4. Doanh nghiệp đáp ứng tốt nhu cầu của khách hàng & 0,722 \\
\hline TRUST5. Doanh nghiệp sẽ giải quyết trong trường hợp sản phẩm có vấn đề & 0,551 \\
\hline \multicolumn{2}{|l|}{ Trách nhiệm thương mại (CR) } \\
\hline CR1. Doanh nghiệp sáng tạo và liên tục giới thiệu sản phẩm mới ra thị trường & 0,784 \\
\hline CR2. Luôn nố lực cải thiện sản phầm và dịch vụ cho khách hàng & 0,848 \\
\hline CR3. Luôn cung cấp sản phẩm và dịch vụ có chất lượng cao & 0,771 \\
\hline CR4. Doanh nghiệp thông báo một cách chính xác và trung thực về các đặc điểm của sản phẩm & Loại \\
\hline CR5. Doanh nghiệp giải quyết các khiếu nại của khách hàng một cách nhanh chóng & 0,834 \\
\hline CR6. Hoạt động quảng cáo/khuyến mãi của doanh nghiệp trung thực & 0,829 \\
\hline \multicolumn{2}{|l|}{ Trách nhiệm đạo đức (ER) } \\
\hline $\begin{array}{l}\text { ER1. Doanh nghiệp quan tâm thực hiện đầy đủ nghĩa vụ đối với các bên liên quan (Đại lý, nhà } \\
\text { cung cấp, khách hàng...) }\end{array}$ & Loại \\
\hline ER2. Doanh nghiệp quan tâm đến quyền con người khi thực hiện các hoạt động kinh doanh & 0,765 \\
\hline $\begin{array}{l}\text { ER3. Doanh nghiệp luôn tôn trọng các chuần mực theo quy định của pháp luật khi thực hiện hoạt } \\
\text { động kinh doanh }\end{array}$ & 0,809 \\
\hline $\begin{array}{l}\text { ER4. Doanh nghiệp tôn trọng các nguyên tắc đạo đức trong các mối quan hệ hơn việc đạt được } \\
\text { hiệu quả kinh tế cao }\end{array}$ & 0,797 \\
\hline
\end{tabular}


ĐỐI VỚI HOẠT ĐỘNG TRÁCH NHIỆM XÃ HỘI DOANH NGHIỆP

\begin{tabular}{|l|c|}
\hline Trách nhiệm xã hội (SR) & 0,556 \\
\hline SR1. Doanh nghiệp quan tâm đến việc bảo vệ môi trường tự nhiên & 0,702 \\
\hline $\begin{array}{l}\text { SR2. Doanh nghiệp sử dụng một phần ngân sách để đóng góp cho xã hội và ủng hộ những hoàn } \\
\text { cảnh khó khăn }\end{array}$ & 0,739 \\
\hline SR3. Doanh nghiệp hố trợ sự phát triên của xã hội, tài trợ các hoạt động văn hóa/xã hội & 0,710 \\
\hline SR4. Doanh nghiệp quan tâm cải thiện mức sông chung của xã hội & 0,860 \\
\hline Thái độ của khách hàng đối với hoạt động trách nhiệm xã hội doanh nghiệp (CA) & 0,814 \\
\hline CA1. Tôi xem việc doanh nghiệp thực hiện trách nhiệm xã hội là điều tốt & 0,882 \\
\hline CA2. Tôi thích các doanh nghiệp thực hiện trách nhiệm xã hội & \\
\hline CA3. Tôi có thái độ thuận lợi đối với các doanh nghiệp thực hiện trách nhiệm xã hội & \\
\hline Phương sai trích: 68,9\% & \\
\hline
\end{tabular}

(Nguồn: Kết quả xủ lý số liệu trên phần mềm SPSS 24.0)

Bảng 2 cho thấy các biến trong mô hình nghiên cứu được trích thành 8 nhân tố có phương sai trích là $68,9 \%>50 \%$. Trọng số nhân tố của các biến đo lường đều lớn hơn 0,5 và chênh lệch giữa các trọng số lớn hơn 0,3 . Như vậy, các biến đều đạt giá trị hội tụ.

\subsubsection{Phân tích nhân tố khẳng định (CFA)}

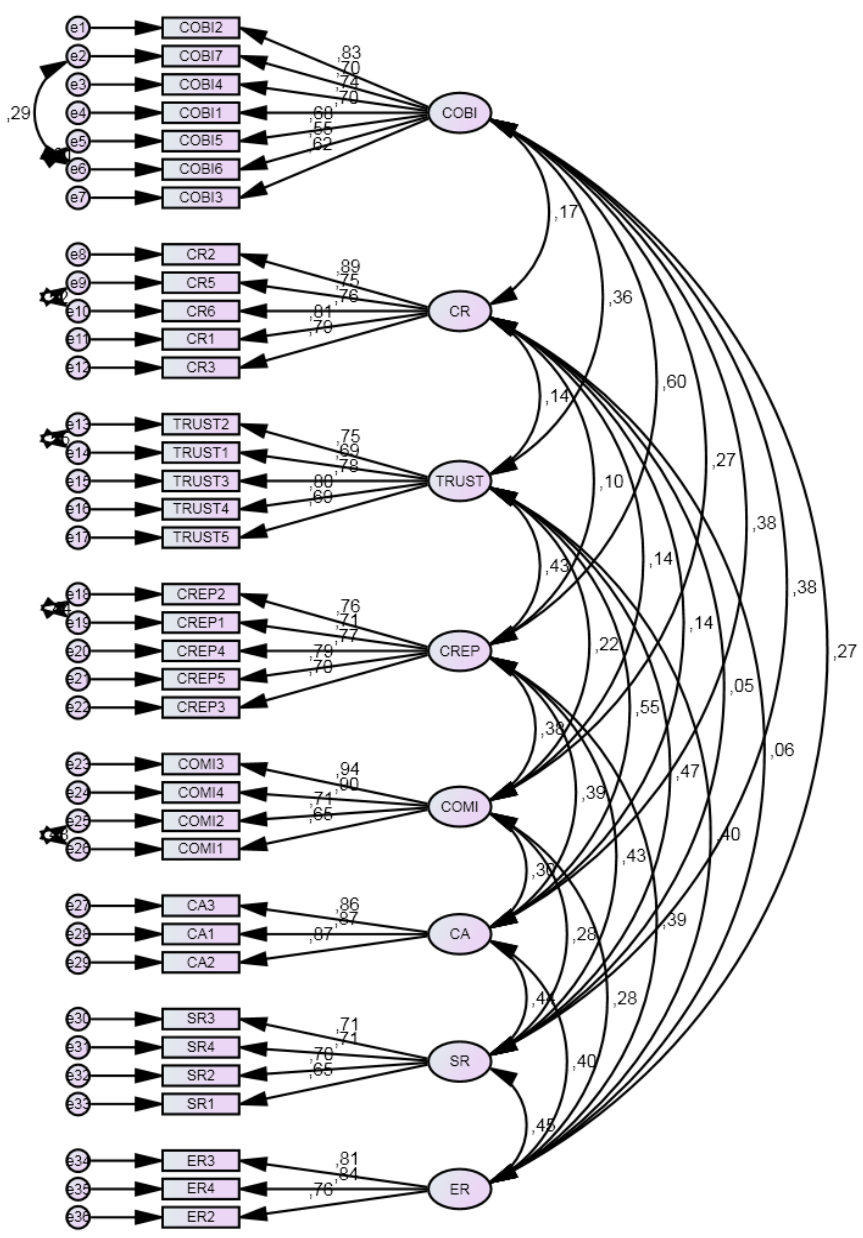

Chi-square $=1.169,2 ; \mathrm{df}=560 ; \mathrm{P}=0,000$

Chi-square/df $=2,088 ;$ GFI $=0,904 ;$ TLI $=0,946$

$\mathrm{CFI}=0,952 ; \mathrm{RMSEA}=0,042$

Hình 2. Mô hình CFA tới hạn (chuẩn hóa) 
Mô hình tới hạn được sử dụng để đánh giá tất cả các khái niệm trong mô hình. Kết quả cho thấy có 560 bậc tự do và mô hình này thích hợp với dữ liệu thị trường (Chi-square/df $=2,088<5 ; \mathrm{GFI}=0,904\rangle$ $0,9 ; \mathrm{TLI}=0,946>0,9 ; \mathrm{CFI}=0,952>0,9$ và $\mathrm{RMSEA}=0,042<0,08)$ (Kettinger và Lee, 1995). Các thang đo trách nhiệm xã hội $(\mathrm{CR})$, trách nhiệm đạo đức $(\mathrm{ER})$ và thái độ của khách hàng $(\mathrm{CA})$ không có tương quan giữa các sai số đo lường nên các biến quan sát đạt được tính đơn hướng, các thang đo hình ảnh nước xuất xứ thương hiệu (COBI), trách nhiệm thương mại (CR), danh tiếng doanh nghiệp (CREP), hình ảnh nước sản xuất (COMI) và niềm tin của khách hàng (TRUST) không đạt tính đơn hướng. Các trọng số chuẩn hóa của các biến quan sát giao động từ $0,55-0,92$ đều đạt yêu cầu (lớn hơn 0,5 ) và các trọng số chưa chuẩn hóa đều có ý nghĩa thống kê $(\mathrm{P}=0,00)$ với độ tin cậy $95 \%$, nên các biến quan sát dùng để đo lường các khái niệm đạt giá trị hội tụ. Ngoài ra, hệ số tương quan của từng cặp khái niệm khác biệt so với 1 có ý nghĩa thống kê nên các thành phần đạt giá trị phân biệt (Hình 2).

Kết quả kiểm định độ tin cậy và phương sai trích của các khái niệm cho thấy, độ tin cậy Cronbach's alpha và độ tin cậy tổng hợp của các thành phần đều lớn hơn 0,6 và phương sai trích lớn hơn 0,5 . Như vậy các thang đo đều đạt độ tin cậy cao.

\section{3. Ước lượng các mối quan hệ cấu trúc}

\subsubsection{Kiểm định mô hình lí thuyết bằng SEM}

Kết quả kiểm định mô hình cấu trúc được trình bày ở hình 3 cho thấy mô hình có 572 bậc tự do với Pvalue $=0,00<0,05$. Các chỉ số đều phù hợp với dữ liệu thị trường (Chi-quare/df < 5; GFI, CFI, TLI > 0,9, RMSEA $<0,8)$.

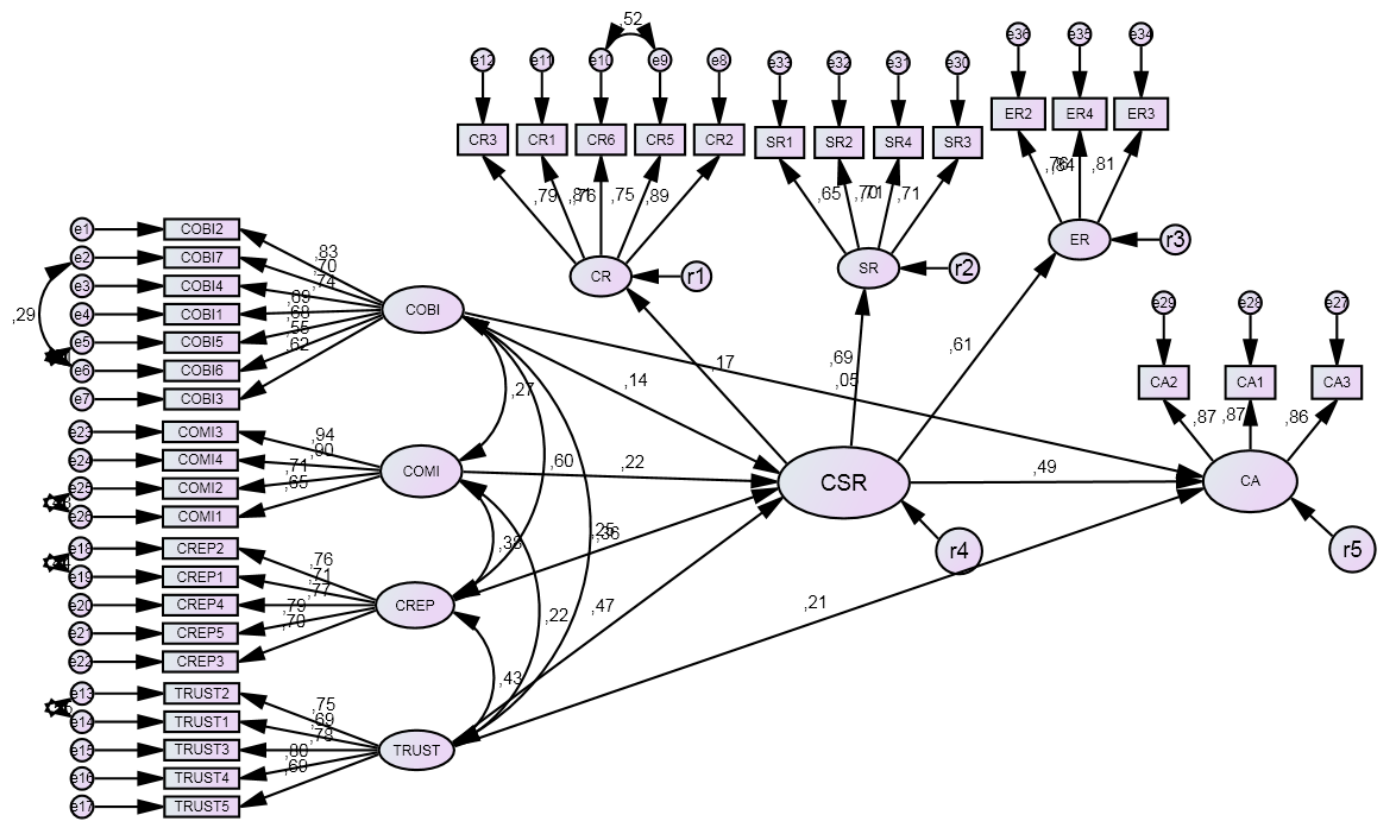

$$
\begin{aligned}
\text { Chi-square } & =1.189,14 ; \mathrm{df}=572 ; \mathrm{P}=0,000 \\
\text { Chi-square } / \mathrm{df} & =2,079 ; \text { GFI }=0,903 ; \text { TLI }=0,951 \\
\text { CFI } & =0,951 ; \text { RMSEA }=0,042
\end{aligned}
$$

Hình 3. Kết quả phân tích SEM mô hình lí thuyết (đã chuẩn hóa)

Kết quả ước lượng các tham số chính của mô hình được trình bày trong bảng 3 . Kết quả cho thấy, tác động của hình ảnh nước xuất xứ thương hiệu đến thái độ của khách hàng không có ý nghĩa thống kê $(\mathrm{P}=0,356>0,05$ với mức ý nghĩa $\alpha=5 \%)$. Các mối quan hệ còn lại trong mô hình đều có ý nghĩa thống kê với mức ý nghĩa $\alpha=5 \%$. Như vậy, các giả thuyết $\mathrm{H}_{1}, \mathrm{H}_{2 \mathrm{a}}, \mathrm{H}_{3}, \mathrm{H}_{4}, \mathrm{H}_{5 \mathrm{a}}, \mathrm{H}_{5 \mathrm{~b}}$ được chấp nhận. 
ĐỐI VỚI HOẠT ĐộNG TRÁCH NHIỆM XÃ HỘI DOANH NGHIỆP

Bảng 3. Hệ số hồi quy các mối quan hệ trong mô hình lý thuyết

\begin{tabular}{|c|c|c|c|c|c|c|}
\hline $\begin{array}{c}\text { Giả } \\
\text { thuyết }\end{array}$ & Mối quan hệ & $\begin{array}{c}\text { Hệ số chưa } \\
\text { chuẩn hóa }\end{array}$ & $\begin{array}{c}\text { Hệ số } \\
\text { chuẩn } \\
\text { hóa }\end{array}$ & SE & CR & P-value \\
\hline $\mathrm{H}_{1}$ & $\begin{array}{c}\text { Nhận thức CSR } \rightarrow \text { Thái độ } \\
\text { khách hàng }\end{array}$ & 0,974 & 0,487 & 0,205 & 4,743 & 0,00 \\
\hline $\mathrm{H}_{2 \mathrm{a}}$ & $\begin{array}{c}\text { Hình ảnh nước xuất xứ } \\
\text { thương hiẹ̣u } \rightarrow \text { Nhận thức } \\
\text { CSR }\end{array}$ & 0,114 & 0,139 & 0,055 & 2,083 & 0,037 \\
\hline $\mathrm{H}_{2 \mathrm{~b}}$ & $\begin{array}{c}\text { Hình ảnh nước xuất xứ } \\
\text { thương hiệu } \rightarrow \text { Thái độ } \\
\text { khách hàng }\end{array}$ & 0,083 & 0,051 & 0,090 & 0,923 & 0,356 \\
\hline $\mathrm{H}_{3}$ & $\begin{array}{c}\text { Hình ảnh nước sản xuất } \rightarrow \\
\text { Nhận thức CSR }\end{array}$ & 0,103 & 0,219 & 0,024 & 4,233 & 0,00 \\
\hline $\mathrm{H}_{4}$ & $\begin{array}{c}\text { Danh tiếng } \rightarrow \text { Nhận thức } \\
\text { CSR }\end{array}$ & 0,189 & 0,246 & 0,055 & 3,463 & 0,00 \\
\hline $\mathrm{H}_{5 \mathrm{a}}$ & $\begin{array}{c}\text { Niềm tin } \rightarrow \text { Nhận thức } \\
\text { CSR }\end{array}$ & 0,339 & 0,473 & 0,048 & 7,108 & 0,00 \\
\hline $\mathrm{H}_{5 \mathrm{~b}}$ & $\begin{array}{c}\text { Niềm tin } \rightarrow \text { Thái độ của } \\
\text { khách hàng }\end{array}$ & 0,295 & 0,205 & 0,104 & 2,820 & 0,00 \\
\hline
\end{tabular}

(Nguồn: Kết quả xủ lý số liệu trên phần mềm Amos 20)

\subsubsection{Phân tích biến trung gian}

Nghiên cứu vai trò trung gian của nhận thức CSR trong mối quan hệ giữa các biến độc lập (Hình ảnh nước xuất xứ thương hiệu và niềm tin của khách hàng) và biến phụ thuộc (Thái độ của khách hàng) là mục tiêu quan trọng của nghiên cứu. Để xác định vai trò trung gian có ý nghĩa, bốn điều kiện cần đáp ứng: (1) Các biến độc lập (Hình ảnh nước xuất xứ thương hiệu và niềm tin của khách hàng) tác động có ý nghĩa đến nhận thức CSR; (2) Nhận thức CSR tác động có ý nghĩa đến thái độ của khách hàng ; (3) Các biến độc lập tác động có ý nghĩa đến thái độ của khách hàng; và (4) Tác động của các biến độc lập đến biến thái độ của khách hàng không có ý nghĩa thống kê hoặc giảm khi tính toán tác động của biến trung gian (Andrews \& cộng sự, 2004).

Bảng 4. Kết quả kiểm định mô hình

\begin{tabular}{|c|c|c|c|c|c|c|c|c|}
\hline Mô hình & $\chi^{2}$ & df & $\overline{\chi^{2} / \mathrm{df}}$ & $\mathbf{P}$ & GFI & TLI & CFI & RMSEA \\
\hline Mô hình 1 & 757,4 & 313 & 2,42 & 0,00 & 0,915 & 0,944 & 0,950 & 0,048 \\
\hline Mô hình 2 & 331,3 & 84 & 3,94 & 0,00 & 0,932 & 0,938 & 0,950 & 0,069 \\
\hline Mô hình 3 & 753,2 & 311 & 2,42 & 0,00 & 0,915 & 0,944 & 0,950 & 0,048 \\
\hline \multicolumn{3}{|c|}{ Mối quan hệ } & \multicolumn{2}{|c|}{ Mô hình 1} & \multicolumn{2}{|c|}{ Mô hình 2} & \multicolumn{2}{|c|}{ Mô hình 3} \\
\hline \multicolumn{3}{|c|}{$\begin{array}{l}\text { Hình ảnh nước xuất xứ thương hiệu } \\
\rightarrow \text { Nhận thức CSR }\end{array}$} & \multicolumn{2}{|c|}{$0,310 * * *$} & & & \multicolumn{2}{|c|}{$0,310 * * *$} \\
\hline \multicolumn{3}{|c|}{$\begin{array}{c}\text { Niềm tin của khách hàng } \rightarrow \text { Nhận } \\
\text { thức CSR }\end{array}$} & \multicolumn{2}{|c|}{$0,633 * * *$} & & & \multicolumn{2}{|c|}{$0,559 * * *$} \\
\hline \multicolumn{3}{|c|}{$\begin{array}{c}\text { Nhận thức CSR } \rightarrow \text { Thái độ khách } \\
\text { hàng }\end{array}$} & \multicolumn{2}{|c|}{$0,174 * * *$} & & & \multicolumn{2}{|c|}{$0,471 * * *$} \\
\hline \multicolumn{3}{|c|}{$\begin{array}{l}\text { Hình ảnh nước xuất xứ thương hiệu } \\
\rightarrow \text { Thái độ khách hàng }\end{array}$} & & & \multicolumn{2}{|c|}{$0,203 * * *$} & \multicolumn{2}{|l|}{0,057} \\
\hline \multicolumn{3}{|c|}{$\begin{array}{c}\text { Niềm tin của khách hàng } \rightarrow \text { Thái độ } \\
\text { khách hàng }\end{array}$} & & & \multicolumn{2}{|c|}{$0,481 * * *$} & \multicolumn{2}{|c|}{$0,217 * * *$} \\
\hline
\end{tabular}


Hai điều kiện đầu tiên được kiểm tra bằng cách ước lượng mô hình 1 (Bảng 4). Kết quả cho thấy mô hình phù hợp với dữ liệu thị trường và các mối quan hệ có ý nghĩa thống kê với mức ý nghĩa 0,01 , nên điều kiện 1 và 2 được đáp ứng. Điều kiện 3 được kiểm tra trong mô hình 2 , kết quả mô hình phù hợp với dữ liệu thị trường và các mối quan hệ có ý nghĩa thống kê với mức ý nghĩa 0,01 , nên điều kiện 3 được đáp ứng. Cuối cùng, so sánh giữa mô hình 2 và mô hình 3 (Bảng 4) cho thấy tác động của hình ảnh nước xuất xứ thương hiệu đến thái độ của khách hàng không có ý nghĩa thống kê khi tính toán tác động của biến trung gian nhận thức $\mathrm{CSR}$, tác động của niềm tin của khách hàng đến thái độ của khách hàng giảm xuống khi tính toán tác động của biến trung gian nhận thức CSR $(0,217$ so với 0,481$)$. Như vậy nhận thức CSR là trung gian hoàn toàn (Full mediation) tác động của hình ảnh nước xuất xứ thương hiệu đến thái độ của khách hàng, đồng thời nhận thức CSR là trung gian một phần (Partial mediation) tác động của niềm tin đến thái độ của khách hàng.

\section{THẢO LUẬn KẾT QUẢ VÀ HÀM Ý QUẢN TR!}

\subsection{Thảo luận kết quả nghiên cứu}

Nghiên cứu này nhằm mục đích khám phá sự hiểu biết hơn nữa về các yếu tố ảnh hưởng đến nhận thức và thái độ của khách hàng đối với hoạt động trách nhiệm xã hội doanh nghiệp.

Kết quả cho thấy rằng, hình ảnh nước xuất xứ được xem xét ở hai khía cạnh (Hình ảnh nước xuất xứ thương hiệu và hình ảnh nước sản xuất) đều có ảnh hưởng trực tiếp đến nhận thức CSR. Như vậy, không chỉ hình ảnh nước xuất xứ thương hiệu có ảnh hưởng tích cực đến nhận thức CSR, mà việc lựa chọn nước sản xuất sản phẩm cũng ảnh hưởng đến nhận thức CSR. Kết quả này phù hợp với nghiên cứu của Han (2015) [14] cho rằng, người dân Hàn Quốc kỳ vọng các doanh nghiệp đến từ Châu âu sẽ thực hiện trách nhiệm xã hội doanh nghiệp tốt hơn các doanh nghiệp Hàn Quốc. Ngoài ra, danh tiếng của doanh nghiệp cũng ảnh hưởng trực tiếp đến nhận thức CSR. Khi doanh nghiệp có danh tiếng tốt thì khách hàng nhận thức các hoạt động trách nhiệm xã hội của doanh nghiệp theo chiều hướng tích cực. Kết quả nghiên cứu này phù hợp với nghiên cứu của Shim và Yang (2016) [5] khi cho rằng doanh nghiệp nên thực hiện các hoạt động CSR khi có danh tiếng tốt. Cuối cùng, một trong những yếu tố quan trọng ảnh hưởng tích cực đến nhận thức CSR là niềm tin của khách hàng. Khi khách hàng tin tưởng doanh nghiệp, họ sẽ nhận thức các hoạt động CSR theo chiều hướng tích cực. Nghiên cứu này đã bổ sung bằng chứng thực nghiệm quan trọng từ những gợi ý của các nghiên cứu trước.

Trong các yếu tố ảnh hưởng đến thái độ của khách hàng đối với hoạt động CSR, nhận thức CSR và niềm tin của khách hàng có ảnh hưởng trực tiếp đến thái độ của khách hàng. Hình ảnh nước xuất xứ thương hiệu không có ảnh hưởng trực tiếp đển thái độ của khách hàng. Tuy nhiên, hình ảnh nước xuất xứ thương hiệu có ảnh hưởng gián tiếp đến thái độ của khách hàng thông qua trung gian hoàn toàn là nhận thức CSR. Điều này cho thấy, hình ảnh nước xuất xứ thương hiệu có thể ban đầu tạo ra những nhận thức tích cực về các hoạt động CSR trước khi ảnh hưởng đến thái độ của khách hàng. Ngoài ra, niềm tin cũng có ảnh hưởng gián tiếp đến thái độ của khách hàng thông qua trung gian một phần là nhận thức CSR. Kết quả này phù hợp một phần với nghiên cứu của Ellen và cộng sự (2006) [20] khi cho rằng, hoạt động CSR ảnh hưởng tích cực đến thái độ của khách hàng.

Xét về mức độ ảnh hưởng, trong các yếu tố có ảnh hưởng đến nhận thức CSR, niềm tin có ảnh hưởng mạnh nhất, sau đó là danh tiếng, hình ảnh nước sản xuất và hình ảnh nước xuất xứ thương hiệu. Điều này cho thấy vai trò quan trọng của việc xây dựng niềm tin của khách hàng cũng như xây dựng danh tiếng doanh nghiệp. Trong các yếu tố ảnh hưởng đến thái độ của khách hàng, nhận thức CSR có ảnh hưởng mạnh nhất, tiếp theo là niềm tin của khách hàng và cuối cùng là hình ảnh nước xuất xứ thương hiệu.

\subsection{Hàm ý quản trị}

Hiện nay, việc thực hiện các hoạt động trách nhiệm xã hội đã được sự quan tâm ngày càng lớn của các doanh nghiệp. Các doanh nghiệp thực hiện CSR với mong muốn nâng cao hiệu quả hoạt động kinh doanh của doanh nghiệp. Tuy nhiên, có thể việc thực hiện CSR sẽ không dẫn đến hiệu quả kỉnh doanh tích cực, vì khách hàng xem các hoạt động CSR là hoạt động tư lợi vì mục đích của doanh nghiệp [5]. Như vậy, việc nghiên cứu các yếu tố ảnh hưởng đến nhận thức và thái độ của khách hàng đối với CSR có vai trò quan 
trọng trong cả lý thuyết và thực tiễn kinh doanh để nâng cao nhận thức và thái độ của khách hàng đối với hoạt động CSR.

Để tăng cường nhận thức CSR của khách hàng, các nghiên cứu trước đây khuyến nghị các doanh nghiệp nên xem xét vai trò của danh tiếng doanh nghiệp [31] [5] và nước xuất xứ thương hiệu [14]. Kết quả của nghiên cứu này cho thấy rằng, danh tiếng doanh nghiệp và hình ảnh nước xuất xứ thương hiệu đều có vai trò quan trọng trong việc nâng cao nhận thức CSR của khách hàng. Bên cạnh đó, nghiên cứu này cũng khám phá hai yếu tố quan trọng có ảnh hưởng đến nhận thức CSR là hình ảnh nước sản xuất và niềm tin của khách hàng. Những khám phá này có vai trò quan trọng đối với các nhà quản lý trong việc phân bổ các nguồn lực hạn chế. Như vậy, không phải lúc nào doanh nghiệp cũng được hưởng lợi khi thực hiện hoạt động CSR, ngay cả khi doanh nghiệp đầu tư nguồn lực lớn cho các hoạt động CSR. Bằng chứng của nghiên cứu này cho thấy, khi doanh nghiệp có danh tiếng xấu và khách hàng không tin tưởng doanh nghiệp thì nhận thức CSR của khách hàng sẽ giảm xuống đáng kể. Ngoài ra, nếu hình ảnh nước xuất xứ thương hiệu và hình ảnh nước sản xuất kém thuận lợi thì cũng ảnh hưởng đến nhận thức CSR. Vì vậy, ngoài việc quan tâm thực hiện các sáng kiến trách nhiệm xã hội doanh nghiệp, các doanh nghiệp ô tô tại Việt Nam cần phải tập trung nâng cao danh tiếng cũng như phát triển niềm tin của khách hàng. Đồng thời, các doanh nghiệp có chiến lược lựa chọn nước sản xuất phù hợp và có những chính sách truyền thông phù hợp để nâng cao ảnh hưởng của nước xuất xứ nếu nó thuận lợi và ngược lại.

Thái độ của khách hàng đối với các hoạt động CSR có vai trò quan trọng đối với các doanh nghiệp trong việc thực hiện các sáng kiến CSR. Nghiên cứu này cho thấy khách hàng có thái độ tích cực đối với hoạt động CSR khi nhận thức CSR của khách hàng tích cực, đồng thời họ có niềm tin đối với doanh nghiệp. Như vậy, việc xây dựng niềm tin của khách hàng có vai trò rất quan trọng ảnh hưởng đến cả nhận thức và thái độ của khách hàng đối với hoạt động CSR.

\subsection{Hạn chế và hướng nghiên cứu tiếp theo}

Đầu tiên, do hiệu ứng hào quang làm cho đo lường CSR có thể có nhiều sai lệch, vì vậy việc sử dụng các phương pháp nghiên cứu khác nhau để đánh giá khái niệm này sẽ tăng cường độ tin cậy của kết quả nghiên cứu. Ngoài ra, nghiên cứu này thực hiện trong ngành ô tô tại Việt Nam, các nghiên cứu tiếp theo có thể thực hiện trong các ngành khác để kiểm định tính phổ biến của đo lường.

Thứ hai, nghiên cứu này xem xét khái niệm CSR là khái niệm tổng hợp gồm 3 nhân tố, tuy nhiên một số nghiên cứu trước cho rằng, các bên liên quan nhận thức khác nhau về các thành phần riêng biệt. Vì vậy, việc kiểm tra tác động của các thành phần riêng biệt trong khái niệm CSR nên thực hiện trong tương lai.

\section{TÀI LIỆU THAM KHẢO}

[1] T. Brown and P. Dacin, "The company and the product: corporate associations and consumer product responses," Journal of Marketing , vol. 6, no. 1, pp. 68-84, 1997.

[2] G. Muller-Christ, Sustainable management: Coping with the dilemmas of resource-oriented management, Springer Science \& Business Media, 2011.

[3] K. L. Ailawadi, S. A. Neslin, Y. J. Luan and G. A. Taylor, "Does retailer CSR enhance behavioral loyalty? A case for benefit segmentation," International Journal of Research in Marketing, vol. 31, no. 2, pp. 156-167, 2014.

[4] M. Fatma and Z. Rahman, "Consumer perspective on CSR literature review and future research agenda," Management Research Review, vol. 38, no. 2, pp. 195-216, 2015.

[5] K. Shim and S. Yang, "The effect of bad reputation: The occurrence of crisis, corporate social responsibility, and perceptions of hypocrisy and attitudes toward a company.," Public Relations Review, vol. 42, no. 1, pp. 68$78,2016$. 
[6] C. B. Bhattacharya and S. Sen, "Doing better at doing good: When, why, and how consumers respond to corporate social initiatives," California management review, vol. 47, no. 1, pp. 9-24, 2004.

[7] I. Gigauri, "Attitudes of georgian consumers towards corporate social responsibility," European Scientific Journal, vol. 8, no. 9, 2012.

[8] A. B. Carroll, "A three-dimensional conceptual model of corporate performance," Academy of management review, vol. 4, no. 4, pp. 497-505, 1979.

[9] I. M. Monirul and J. H. Han, "Perceived quality and attitude toward tea \& coffee by consumers," International Journal of Business Research and Management, vol. 3, no. 3, pp. 100-112, 2012.

[10] R. Trudel and J. Cotte, "Does it pay to be good?," MIT Sloan Management Review, vol. 50, no. $2,2009$.

[11] S. Sen, C. Bhattacharya and D. Korschun, "The Role of Corporate Social Responsibility in Strengthening Multiple Stakeholder Relationships: A Field Experiment," Journal of the Academy of Marketing Science, vol. 34, no. 2, pp. 158-166, 2006.

[12] M. S. Roth and J. B. Romeo, "Matching product catgeory and country image perceptions: A framework for managing country-of-origin effects," Journal of international business studies, vol. 23, no. 3, pp. 477-497, 1992.

[13] N. A. Gardberg and D. H. Schepers, "Do stakeholders detect corporate social performance signals?," Academy of Management Proceedings, vol. 2008, no. 1, pp. 1-6, 2008.

[14] C. M. Han, "Consumer expectations of corporate social responsibility of foreign multinationals in Korea," Emerging Markets Finance and Trade, vol. 51, no. 2, pp. 293-305, 2015.

[15] D. Bamber, S. Phadke and A. Jyotishi, "Product-knowledge, ethnocentrism and purchase intention: COO Study in India," Global Markets and Workforce, 2011.

[16] S. Guercini and S. Ranfagni, "Integrating country-of-origin image and brand image in corporate rebranding: the case of China," Marketing Intelligence \& Planning, vol. 31, no. 5, pp. 508-521, 2013.

[17] C. Fombrun, Reputation, John Wiley \& Sons, Ltd, 1996.

[18] J. Bae and G. T. Cameron, "Conditioning effect of prior reputation on perception of corporate giving," Public Relations Review, vol. 32, no. 2, pp. 144-150, 2006.

[19] J. Park, H. Lee and C. Kim, "Corporate social responsibilities, consumer trust and corporate reputation: South Korean consumers' perspectives," Journal of Business Research, vol. 67, no. 3, pp. 295-302, 2014.

[20] P. S. Ellen, D. J. Webb and L. A. Mohr, "Building corporate associations: Consumer attributions for corporate socially responsible programs," Journal of the Academy of Marketing Science, vol. 34, no. 2, pp. 147-157, 2006.

[21] R. C. Mayer, J. H. Davis and F. D. Schoorman, "An integrative model of organizational trust," Academy of management review, vol. 20, no. 3, pp. 709-734, 1995.

[22] D. H. McKnight, V. Choudhury and C. Kacmar, "Developing and validating trust measures for e-commerce: An integrative typology," Information systems research, vol. 13, no. 3, pp. 334-359, 2002. 


\section{CÁC YÊU TỐ TÁC ĐộNG ĐẾN NHẬN THỨC VÀ THÁI ĐỘ CỦA KHÁCH HÀNG ĐỐI VỚI HOẠT ĐộNG TRÁCH NHIỆM XÃ HỘI DOANH NGHIỆP}

[23] J. Singh, M. de los Salmones Sanchez and I. del Bosque, "Understanding Corporate Social Responsibility and Product Perceptions in Consumer Markets: A Cross-Cultural Evaluation," Journal of Business Ethics, vol. 80, no. 3, pp. 579-611, 2008.

[24] Ponzi, L. J., Fombrun, C. J., \& Gardberg, N. A, "RepTrakTM pulse: Conceptualizing and validating a short-form measure of corporate reputation," Corporate Reputation Review, vol. Vol.14, no. Iss.1, pp. 15-35, 2011.

[25] N. Mohd Yasin, M. Nasser Noor and O. Mohamad, "Does image of country-of-origin matter to brand equity?," Journal of Product \& brand management, vol. 16, no. 1, pp. 38-48, 2007.

[26] Y. Chen and Y. Su, "Do country-of-manufacture and country-of-design matter to industrial brand equity?," Journal of Business \& Industrial Marketing, vol. 27, no. 1, pp. 57-68, 2011.

[27] H. T. Keh and Y. Xie, "Corporate reputation and customer behavioral intentions: The roles of trust, identification and commitment," Industrial Marketing Management, vol. 38, no. 7, pp. 732-742, 2009.

[28] S. Sun and Y. Wang, "Examining the role of beliefs and attitudes in online advertising: A comparison between the USA and Romania," International Marketing Review, vol. 27, no. 1, pp. 87-107, 2010.

[29] D. G. Garson, "Factor analysis: statnotes," Retrieved March, p. 22, 2008.

[30] R. A. Peterson, "A meta-analysis of Cronbach's coefficient alpha," Journal of consumer Research, vol. 21, no. 2, pp. 381-403, 1994.

[31] Y. Yoon, Z. Gürhan-Canli and N. Schwarz, "The effect of corporate social responsibility (CSR) activities on companies with bad reputations," Journal of consumer psychology, vol. 16, no. 4, pp. 377-390, 2006.

[32] J. C. Andrews, R. G. Netemeyer, S. Burton, D. P. Moberg and A. Christiansen, "Understanding adolescent intentions to smoke: An examination of relationships among social influence, prior trial behavior, and antitobacco campaign advertising," Journal of Marketing, vol. 68, no. 3, pp. 110-123, 2004.

Ngày nhận bài: 05/04/2018

Ngày chấp nhận đăng: 09/09/2018 